\author{
WOJCIECH DUSZYŃSKI (D), BARTOSZ JAN KOŁOCZEK ID \\ JAGIELLONIAN UNIVERSITY, KRAKÓW
}

\title{
ALCUNI COMMENTI SULLA RICEZIONE DELLA FIGURA DI IFICRATE DI ATENE NELLA LETTERATURA LATINA E OCCIDENTALE ${ }^{1}$
}

ABSTRACT: Ificrate di Atene (c. 420 - c. 350 a.C.) è un comandante militare greco che sembra essere un esempio di una figura famosa nell'antichità, ma quasi completamente dimenticata nei tempi successivi. Ha ricoperto più volte l'incarico di stratega nella sua città natale e nel 390 ha vinto gli Spartani nella battaglia di Lecheo. È stato condottiero al servizio dei sovrani traci e del re di Persia. Molto probabilmente ha apportato innovazioni negli armamenti di fanteria: conosciute come "la riforma di Ificrate". La sua fama è andata ben oltre il mondo greco, come dimostrano i riferimenti conservati nella letteratura latina, sovente molto utili per la ricostruzione della sua biografia. Pertanto, l'Ateniese apparteneva ai personaggi piuttosto noti tra i Romani, che probabilmente attirarono l'attenzione su aspetti notevolmente diversi dal suo ritratto nelle fonti greche. I riferimenti sorprendenti su Ificrate si trovano anche nella letteratura moderna (sia neo-latina, che sviluppata in lingue nazionali). Lo scopo di questo articolo è quello di identificare quegli elementi della biografia del comandante ateniese, che erano di particolare interesse per gli autori latini e moderni basati sulla antica letteratura latina, di determinare le fonti che hanno usato e specificare le ragioni del loro fascino.

KEY WORDS: Ificrate di Atene, storia greca, letteratura latina, Lucio Ampelio, Michele Marullo Tarcaniota, Pierre de Marivaux, Miles Gloriosus

Questo articolo è stato realizzato nell'ambito del progetto di ricerca n. 2015/17/N/ HS3/00014 finanziato dal National Science Center (Polonia). 
Ificrate, figlio di Timoteo di Atene (del demo di Ramnunte) non è un personaggio particolarmente conosciuto nel mondo moderno. La figura di questo comandante militare della prima metà del IV secolo a.C. appare quasi esclusivamente in parecchi studi specialistici dedicati a vari aspetti della storia di Atene nel periodo classico ${ }^{2}$. Tuttavia, nell'antichità, come possiamo presumere dalle opinioni espresse dagli autori greci, Ificrate godeva di una fama molto maggiore. Il suo contemporaneo Senofonte nelle sue Elleniche lo rende uno degli esempi di buon comandante, dedicando molta attenzione all'organizzazione della sua spedizione a Corcira nel 372 (Xen. Hell. VI 4, 13-35)3. A sua volta, Diodoro Siculo lo menziona tra le persone più importanti della sua epoca (XV 88, 2). Con un tono simile, di Ificrate gran comandante, parla Plutarco, che cita Epaminonda affermando che è difficile decidere chi sia il duce migliore: lui stesso, cioè Epaminonda, Ificrate o il suo concittadino Cabria (Mor. 187a). Come autorità militare la figura di Ificrate appare anche nella Vita dell'imperatore Galba, che inizia con una delle dichiarazioni attribuite al capo ateniese $e^{4}$. Anche le affermazioni di Plutarco dimostrano il grande significato che è stato associato alla sua opinione centinaia di anni dopo la sua morte .

2 L'unico studio monografico su Ificrate è il lavoro di K. Rehdantz (1845), in cui la figura del comandante ateniese fu discussa insieme ad altri capi di questo periodo: Cabria e Timoteo. Sfortunatamente, questo studio può essere considerato obsoleto. Tra le opere più recenti troviamo quasi esclusivamente note enciclopediche (Kirchner 1901; Kahrstedt 1916) e singoli articoli (Bianco 1997; Moreno Hernández 2002). Molta attenzione a Ificrate hanno dedicato anche H.W. Parke (1933: 50-56, 74-82) e M. Bettali (2013: 91-93, 111-128, 374-376) nelle loro opere sul fenomeno del mercenario in Grecia, così come W.K. Pritchett nel secondo volume di The Greek State at War (1974: 62-72, 117-125), W. Lengauer nei suoi studi sui comandanti greci dell'età classica (1979: 110-114) e M.A. Sears in un lavoro più ampio sulla politica di Atene in Tracia (2013: 118-135). Anche il problema delle riforme militari attuate da Ificrate ha ricevuto una separata pubblicazione collettiva (Burliga, Sekunda 2014).

3 Sull'atteggiamento del Senofonte nei confronti di Ificrate vedi Westlake 1978: 207; Lengauer, 1979: 112-114; Tuplin 1993: 72-73, 115-116, 161-162; Toalster 2011: 201; Bianco 2011: 40-46.

$4 \quad$ Plut. Galb. 1, 1. Cfr. Plut., De fort. 5.

5 Sfortunatamente non abbiamo nulla da suggerire che Plutarco abbia dedicato a Ificrate una biografia separata. Tuttavia, a causa della conoscenza incompleta della sua produzione letteraria è difficile escludere completamente tale possibilità. È noto che gran parte del corpus delle opere di Plutarco (tra l'altro, anche la Vita di Epaminonda) 
La figura di Ificrate era familiare anche ai Romani. Inoltre, ha ricevuto numerosi riferimenti interessanti (o addirittura sorprendenti) nella letteratura moderna. Lo scopo di questo articolo è quello di identificare quegli elementi della biografia del comandante ateniese, che erano di particolare interesse per gli autori latini e moderni basati sulla antica letteratura latina, di determinare le fonti che hanno usato e specificare le ragioni del loro fascino.

All'inizio, vale la pena di presentare brevemente la biografia di Ificrate, che possiamo ricostruire sulla base di vari riferimenti nelle fonti. Molto probabilmente, l'Ateniese nacque negli ultimi vent'anni del V secolo a.C., se si crede agli autori antichi, nella famiglia di un sem-

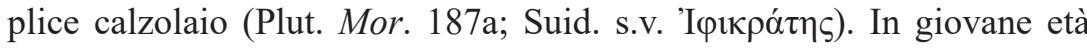
fece dell'attività militare la sua principale occupazione e già prima dei 30 anni diventò il comandante del corpo ateniese di peltasti mercenari

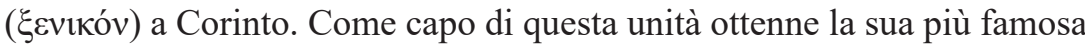
vittoria nella battaglia del Lecheo (390), dove riuscì a sorprendere e distruggere l'intero reggimento (mora) degli opliti spartani (Xen. Hell. IV

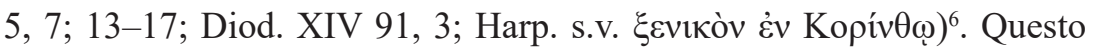
successo in seguito ha portato alla onorificenza, da parte dei concittadini, di un monumento con una dedica personale, eretto a spese pubbliche davanti all'ingresso al Partenone (Dem. XXIII 130) ${ }^{7}$. Eletto come stratega almeno una dozzina di volte, combatté in tutti i maggiori conflitti a cui la sua città partecipò nella prima metà del IV secolo, prima di tutto alla guerra di Corinto (395-387/386), guerra beotica (379-371) e guerra sociale $(357-355)^{8}$.

non è sopravvissuta ai nostri tempi. È difficile decidere se conosciamo tutti i titoli delle sue opere. Il cosiddetto Catalogo di Lampria elenca 227 diverse opere di Plutarco, ma non trasmette i titoli delle singole vite. Inoltre questa raccolta non è completa, in quanto mancano 18 scritti conservati e 15 scritti non salvati. Nondimeno, Plutarco rende Ificrate l'autore di alcuni detti famosi in una collezione a loro dedicata (Detti di re e condottieri), che riguardano principalmente questioni relative ai militari.

$6 \quad$ Un'analisi completa di questa battaglia è presentata in Konceny 2014: 8-42.

$7 \quad$ Vedi Kahrstedt 1916: 2021; Bianco 1997: 194-196. Pausania (I 24, 7) osserva, che l'unico altro personaggio il cui monumento poteva essere visto ai suoi tempi nel Partenone era l'imperatore Adriano. Forse a causa di questa insolita combinazione Pausania si sente obbligato a caratterizzare Ificrate come colui, che ha compiuto "molte

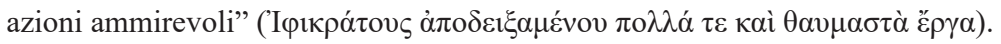

$8 \quad$ Kahrstedt 1916: 2019-221; Bettali 2013: 91-93. 
Negli intervalli tra le guerre condotte dai compatrioti Ificrate cercava "lavoro" all'estero, specialmente nel nord del Mar Egeo. In Tracia entrò nella famiglia del re degli Odrisi Seute II e suo figlio Cotys, sposando la figlia del primo e la sorella del secondo. Come afferma Demostene (XXIII 136), queste connessioni portarono Ificrate di fronte a una situazione problematica, quando nella seconda metà degli anni '60 i rapporti tra Cotys e Atene si interruppero'. Probabilmente Ificrate inizialmente sostenne suo cognato, ma quando lui si trovò nello stato di guerra con i suoi compatrioti, si ritirò a Lesbo. Secondo Eschine (Aesch. II 28), il comandante ateniese fu anche adottato dal re di Macedonia Aminta III (probabilmente a cavallo tra gli anni ' 80 e '70). Le circostanze di questo evento sono purtroppo sconosciute ${ }^{10}$, ma di conseguenza, Ificrate divenne il fratello adottivo del successivo re Filippo II.

La morte di Ificrate avvenne probabilmente alla fine degli anni ' 50 del IV secolo a.C. ${ }^{11}$ Secondo Cornelio Nepote, il comandante morì ad Atene in età avanzata come una persona rispettata dai suoi concittadini. Nella sua vita, verosimilmente non ha ottenuto grandi vittorie in battaglie generali, mentre autori antichi riportano i suoi numerosi successi in schermaglie. Questo è ben combinato con le testimonianze della sua abilità nell'inventare stratagemmi. Secondo Diodoro Siculo, Ificrate ha anche mostrato un talento particolare nel campo dell'innovazione negli armamenti. Di conseguenza (prima di tutto a causa dell'allungamento della lancia e riduzione degli scudi), fu creato un guerriero simile al successivo picchiere macedone (sarissophoros). Non c'è accordo tra gli studiosi sulla forma e il tempo di attuazione di questa cosiddetta riforma di Ificrate, ma la maggior parte di loro la colloca negli anni 378-373,

\footnotetext{
$9 \quad$ Vedi Kallet 1983: 244-251; Harris 1989: 265-271.

10 Secondo Eschine (II 28), Euridice, la moglie di Aminta III, si riferì all'adozione di Ificrate da parte di suo marito, chiedendo supporto allo stratega ateniese intorno all'anno $369 / 368$. Secondo il suo stesso resoconto, il famoso oratore in seguito ha ricordato a Filippo II le parole di sua madre durante la sua missione diplomatica nel 346. Certo, Demostene avrebbe potuto distorcere il corso degli eventi, ma è improbabile che abbia mancato completamente la verità. La sua versione viene confermata anche da Cornelio Nepote (Iph. 3, 2), il quale menziona i contatti di Ificrate con la famiglia di Aminta. $\mathrm{Su}$ questo argomento vedi Harris 1995: 11-12; Heskel 1997: 11-12; Lengauer 2004: 36-37.
}

11 Rehdantz 1845: 230; Kahrstedt 1916: 2021; Bianco 1997: 205-207. 
durante la permanenza del condottiero al servizio mercenario in Persia ${ }^{12}$. Gli fu affidato il compito di preparare l'esercito del gran re per l'invasione dell'Egitto ribelle. Le sue innovazioni nell'equipaggiamento di fanteria, secondo alcuni studiosi, divennero la base per il nuovo esercito di Filippo II di Macedonia e, grazie alle conquiste di Alessandro Magno, si diffusero in tutto il Mediterraneo orientale ${ }^{13}$.

I suddetti cambiamenti nell'equipaggiamento di fanteria divennero oggetto di interesse da parte di Cornelio Nepote (c. 100 - c. 25 a.C.), che incluse una breve biografia di Ificrate nella sua collezione intitolata $D e$ viris illustribus. Questo piccolo scritto (generalmente classificato come esponente di biografia alessandrina ${ }^{14}$ ), composto esclusivamente da tre capitoli è non solo il primo esempio conservato della tradizione della letteratura latina su Ificrate, ma anche l'unico testo continuo a lui dedicato. Nepote ha riconosciuto Ificrate come uno degli ultimi grandi condottieri di Atene, insieme a Timoteo e Cabria ${ }^{15}$. La ragione per averlo incluso nella collezione dei più grandi comandanti della storia era più la sua conoscenza dell'arte marziale che la portata dei suoi successi, vittorie in battaglie o grandi conquiste (Iph. 1, 1: Iphicrates Atheniensis non tam magnitudine rerum gestarum quam disciplina militari nobilitatus est). La competenza di Ificrate era particolarmente evidente nelle sue innovazioni nell'armamento. Nepote sottolinea la lunga continuità della sua attività militare e la mancanza di gravi fallimenti, in particolare quelli dovuti a propria colpa $(I p h .1,2)$. Evoca anche i suoi rapporti con la casa regnante macedone degli Argeadi. Ificrate, come leggiamo, intervenne armatamente per Euridice, la vedova di Aminta III, che, dopo la morte di suo marito, si rifugiò da lui con i suoi figli minori, i futuri re Perdicca III e Filippo II $(I p h .3,2)$. Questo episodio ci è noto solo dalla

\footnotetext{
12 Anderson 1970: 129-131; Stylianou 1998: 344-345; Sekunda 2014: 127-128.

13 Sekunda 2014: 137-142.

14 Anselm 2004: 51-53.

15 Come notato da S. Anselmo (2004: 61-63, 111), le vite dei tre comandanti sopra menzionati constituiscono una parte chiusa all'interno dell'opera di Nepote, che simboleggia l'ultimo periodo della gloria di Atene, associato alla creazione e all'espansione della Seconda lega delio-attica. Ciò è particolarmente indicato dalla fine dell'ultima vita di Timoteo (Tim. 4, 4): Haec extrema fuit aetas imperatorum Atheniensium, Iphicratis, Chabriae, Timothei, neque post illorum obitum quisquam dux in illa urbe fuit dignus memoria.
} 
menzione dell'oratore ateniese Eschine, secondo il quale il suo concittadino sostenne Euridice nella lotta contro il pretendente al trono, un certo Pausania (II 28). Nepote (Iph. 3, 2) si riferisce direttamente all'autorità dello storico Teopompo di Chio, che non solo era l'autore della storia universale della Grecia (le Elleniche), ma anche, una cosa notevole in questo contesto, dell'opera principalmente dedicata alla storia di Filippo II e la sua dinastia (le Storie filippiche) ${ }^{16}$.

La figura del comandante ateniese appariva anche in un'altra opera dedicata a Filippo II, Historiae Philippicae di Pompeo Trogo, che visse nel periodo augusteo. La sua attenzione è stata probabilmente attratta soprattutto dalla giovane età, in cui Ificrate ha rivelato i suoi eccezionali talenti. Giustino (III sec. d.C.), autore di un sommario di questa perduta opera, menziona Ificrate come il capo che, all'età di appena vent'anni, durante la guerra di Corinto (395-387/6) fu inviato per aiutare i Beoti: Quibus rebus cognitis, Athenienses verentes ne iterum Lacedaemoniis victoribus in pristinam sortem servitutis redigerentur, exercitum contrahunt eumque in auxilium Boeotiorum per Iphicraten, XX quidem annos natum, sed magnae indolis iuvenem, duci iubent. Huius adulescentis supra aetatem virtus admirabilis fuit, nec umquam ante eum Athenienses inter tot tantosque duces aut spei maioris aut indolis maturioris imperatorem habuerunt, in quo non imperatoriae tantum, verum et oratoriae artes fuere (VI 5, 1). Il giovane ateniese, a cui i suoi concittadini, come sottolinea lo storico romano, affidarono in effetti il destino della loro patria, minacciata dalle tendenze imperialiste di Sparta, è elogiato non solo per il coraggio straordinario, l'eccezionale maturità, le capacità di comando, ma anche, cosa particolarmente interessante, per singolari capacità oratorie. Ificrate in queste qualità superava tutti gli altri comandanti ateniesi che probabilmente era il più grande elogio di lui, soprattutto se prendiamo in considerazione il fatto che nessun altro stratega è stato descritto da Pompeo Trogo/Giustino in modo così favorevole e la stessa città di Atene era associata ai più eccezionali oratori greci. Una versione molto simile, ma allo stesso tempo assai abbreviata, la troviamo nell'opera

16 La scrittura di Teopompo fu più ampiamente utilizzata da Nepote nell'opera sulle vite dei comandanti ateniesi del IV secolo. Nella Vita di Cabria (Chab. 3, 2-4) troviamo un frammento senza riferimento, ma identico al brano citato da Ateneo di Naucrati dall'opera di Teopompo (Athen. XII 532b = FGrH $117 \mathrm{~F} 105$ ). 
dello storico tardoantico Paolo Orosio. Anche lui presta una particolare attenzione alla giovane età di Ificrate: at vero Athenienses cum conperissent insperata Lacedaemonios victoria sublevatos, pristinae servitutis, de qua tunc respirare vix coeperant, trepidi metu exercitum contrahunt eumque Boeotiis in auxilium adiungunt, commissum Iphicrati duci, qui adulescens admodum, vixdum annos viginti natus, infirmitatem aetatis maturitate animi muniebat (III 1, 21). Entrambi i brani, indubbiamente riferiti alla stessa tradizione letteraria (è difficile dire se Orosio si fosse basato sul testo di Pompeo Trogo, Giustino o qualcun altro) testimoniano l'alto riconoscimento di Ificrate come un vero leader, il cui talento è stato riconosciuto già in giovane età.

La menzione della giovane età del comandante è abbastanza problematica a causa del probabile limite d'età (usualmente si presume che sia stato di 30 anni $^{17}$ ) per l'eleggibilità a tutte le cariche pubbliche ad Atene, comprese quelle militari. Tuttavia, come sottolinea Giustino, la decisione dei concittadini di Ificrate avrebbe potuto essere influenzata dai suoi eccezionali talenti, inclusa la competenza nell'arte retorica. Senofonte, che descrive la battaglia del Lecheo, chiama Ificrate $\tau \tilde{\omega} \nu \pi \varepsilon \lambda \tau \alpha \sigma \tau \tilde{\omega} \nu$ ó $\rho \chi \omega \nu$, contrastandolo chiaramente con il comandante degli opliti Callia, definito come $\sigma \tau \rho \alpha \tau \eta \gamma o ́ s$ (Hell. IV 5, 13). Pare quindi che Giustino e Orosio avessero ragione sulla giovane età di Ificrate e per questo motivo lui a quel tempo non era uno stratega, ma un ufficiale di grado inferiore. Le informazioni fornite dagli storici romani sulla carriera iniziata da Ificrate così presto possono corrispondere alla menzione di Plutarco (Mor. 187a) e ai commenti di uno scoliasta anonimo all'orazione di Demostene Contro Midia (Schol. ad Dem. XXI 62). Secondo entrambi gli autori greci, Ificrate era un semplice soldato ( $\sigma \tau \rho \alpha \tau \iota \omega ́ \tau \eta \varsigma)$, che, durante una delle battaglie navali, saltò sulla nave nemica e, dopo aver catturato il suo comandante, lo rapì a bordo della sua unità. Grazie a questa impresa ricevette un premio di coraggio ( $\dot{\alpha} \rho \sigma \tau \varepsilon i ́ \alpha)$ e venne eletto stratega. Sfortunatamente nessuna relazione specifica il tempo e il luogo di questa azione. Alla luce della cronologia degli eventi, l'unica potenziale possibilità sembra essere

17 Sul limite dell'età per l'eleggibilità alla carica di strateghi ateniesi vedi Develin 1985. Sulla visione dominante scetticismo esprime W. Lengauer (2002), con cui polemizza M.H. Hansen (2006: 22-25). 
la battaglia di Cnido del $394^{18}$. In questo caso, però, torniamo alla questione del limite dell'età di 30 anni, che Ificrate probabilmente non ha ancora compiuto durante quello scontro.

Come sappiamo, la figura di Filippo II fu oggetto di particolare attenzione da parte di Pompeo Trogo ${ }^{19}$. Forse questa è la chiave per comprendere la presenza di Ificrate nel suo lavoro. Come testimoniano i riferimenti sopra menzionati di Eschino e Nepote, il comandante ateniese era probabilmente associato agli Argeadi (specialmente al padre di Filippo II). In questo contesto è molto interessante anche la notizia di Curzio Rufo (attivo nella prima metà del I secolo d.C.) sul figlio di Ificrate, Ificrate il Giovane (III 13), che serviva come rappresentante ateniese alla corte di Dario III e dopo la battaglia di Issos nel 333, fu catturato dall'esercito del vittorioso Alessandro. Una versione simile può essere trovata nell'Anabasi di Alessandro di Flavio Arriano (II 15), uno storico e politico romano del II secolo di origine greca. Alle informazioni fornite da Curzio Rufo, Arriano aggiunge che, per la memoria di suo padre, il famoso re trattava Ificrate il Giovane con grande rispetto, a differenza dei delegati di alcune altre poleis (ad esempio gli spartani furono incatenati), e quando lui si ammalò e morì, pagò le spese di trasporto del suo corpo ad Atene ${ }^{20}$. Questa informazione è molto ben combinata con i riferimenti degli altri autori sui legami familiari che collegavano Ificrate al nonno di Alessandro, Aminta III. In tutte le opere citate, Ificrate appare nel contesto delle connessioni con gli Argeadi. Possiamo quindi supporre, che questo argomento fosse particolarmente interessante agli autori romani.

Come specialista di trucchi di guerra, Ificrate è ovviamente presente negli Strategemata di Sesto Giulio Frontino (c. 40 - c. 103 d.C.). Quasi tutti gli esempi delle sue astuzie militari, descritti da Frontino, appaiono anche nel lavoro successivo di Polieno ${ }^{21}$. Ificrate è per l'autore greco indiscusso numero uno in questo campo, poiché Polieno descrive ben 63 strategemmi dell'Ateniese, contro i 33 trucchi del re spartano Agesilao e Giulio Cesare (entrambi occupano il secondo posto) e i 32 di Alessandro Magno (terzo posto). Nell'opera di Frontino Ificrate appare nove

18 Bianco 1997: 181. Al contrario Kahrsted (1916: 2019) lascia la lotta come anonima.

19 Alonso-Núñez 1987: 58-59.

$20 \quad$ Habicht 1998: 38.

$21 \quad$ Polyaen. III 9. 
volte, il che è un numero significativo (lo stesso numero di volte vengono riferiti gli strategemmi del famoso re spartano Agesilao), ma non sufficiente per salire "sul podio"22. Tuttora, nella scelta di Frontino non sorprende che molto più esposti siano i capi romani. Di solito le versioni di entrambi gli autori differiscono solo leggermente l'una dall'altra. Non è chiaro da dove Frontino abbia ottenuto le sue informazioni, mentre nel caso di Polieno si suppone che, pur conoscendo la collezione dell'autore latino, abbia lavorato indipendentemente da lui e abbia usato il lavoro di Eforo di Cuma ${ }^{23}$. Forse, quindi, anche il precedente autore romano attingeva le sue notizie da questo storico. L'unico stratagemma presente solo nell'opera di Frontino riguarda la situazione, in cui Ificrate uccide un soldato sorpreso a dormire durante una guardia (Strat. III 12, 2). Naturalmente si tratta di mostrare la severità e la passione per la disciplina del comandante ateniese, che a noi è noto anche da altre fonti, compresa la Vita di Ificrate di Nepote.

La presenza di Ificrate nella biografistica, letteratura storica e professionale (militare) non è sorprendente. Tuttavia ci imbattiamo in riferimenti alla figura dell'Ateniese anche in luoghi abbastanza inaspettati. Uno di questi è il Liber memorialis di Lucio Ampelio, un piccolo manuale compendio del II secolo, un'opera creata per uso scolastico, oltre che per uno studente specifico, un certo Macrino. Liber memorialis non è certamente un'enciclopedia o un trattato scientifico e non rivendica neppure tali ambizioni. Nell'introduzione l'autore si rivolge direttamente al destinatario, riferendosi al suo desiderio veramente infantile ed entusiasta "Dato che vuoi sapere tutto, ho scritto questo «Repetitorio», in modo, che tu sappia cos'è il mondo, cosa sono gli elementi, cosa c'è nel cerchio della terra o cosa fece il genere umano" (Praef.: Volenti tibi omnia nosse scripsi hunc librum memorialem, ut noris, quid sit mundus, quid elementa, quid orbis terrarum ferat, vel quid genus humanum peregerit). Sfortunatamente, non sappiamo se il lavoro di Ampelio fosse servito come un manuale per supportare il processo didattico, o se fosse piuttosto un dono fatto al suo studente alla fine dell'istruzione, ma senza dubbio

22 Front. Strat. I 4, 7; 5, 24; 6, 1; II 1, 5; 5, 42; 12, 4; III 12, 2; IV 7, 23.

23 Bianco 2010: 72-75. 
era destinato a servire una ricapitolazione secondaria del materiale già elaborato piuttosto che all'introduzione di nuove questioni ${ }^{24}$.

Il manuale di Ampelio nella sua versione preservata è composto da 50 capitoli (l'opera non ha parte finale, in diversi capitoli sono evidenti i difetti e danni al testo) divisi in due parti: i capitoli 1-29 dedicati all'astronomia/astrologia, alla geografia e ai rudimenti di storia rispondono a tre domande poste nel prologo: la prima: cos'è il mondo e cosa sono gli elementi? la seconda: cosa c'è nel cerchio terrestre? la terza: cosa fece il genere umano? mentre i capitoli 30-50 approfondiscono le notizie storiche della prima parte. In questa seconda parte tra le figure più importanti della storia greca e romana appare anche Ificrate. Il motivo principale della sua inclusione nel compendio scolastico sono i cambiamenti da lui effettuati nell'equipaggiamento della fanteria. Ampelio afferma brevemente che Ificrate ha reso l'armamento più leggero e più facile da usare (15, 16: Iphicrates, rei militaris peritissimus, qui arma habiliore pondere et modo fecit). In questo modo il grammatico poteva sintetizzare le parole di Cornelio Nepote sull'equipaggiamento degli opliti con scudi più piccoli e armature di lino anziché di bronzo (Iph. 1, 3-4: Cum ante illum imperatorem maximis clipeis, brevibus hastis, minutis gladiis uterentur, ille e contrario peltam pro parma fecit - a quo postea peltastae pedites appellantur -, ut ad motus concursusque essent leviores; hastae modum duplicavit, gladios longiores fecit. Idem genus loricarum et pro sertis atque aenis linteas dedit. Quo facto expeditiores milites reddidit: nam pondere detracto, quod aeque corpus tegeret et leve esset, curavit) ${ }^{25}$. Può darsi che Ampelio si riferisse anche alla tradizione di un nuovo tipo di calzari introdotti dal famoso comandante ateniese in relazione alla sua riforma. Diodoro Siculo afferma che erano leggeri e facili da legare (XV

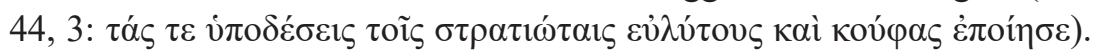
In seguito dal nome dell'inventore furono chiamati gli ificratidi ${ }^{26}$. A que-

24 Vedi Arnaud-Lindet 1993: VII-VIII; 1997: 2302-2303, 2308-2309 e 2312; 1998 : 825-826 e 837-838; König 2010: 7; Bugaeva 2011: 96-98, sebbene già Ducos 1989: 171 e in parte anche Galdi 1922: 80-81 e Terzaghi 1947: 79-92 considerassero il lavoro di Ampelio come un "manuale di scuola". Cfr. anche Kołoczek 2016a: 17; 2016b: 55; 2019: 71-72.

25 Sulle somiglianze tra le Vite di Nepote e brani di Ampelio vedi Wölfflin 1854: 33-34.

26 Vedi Rehdantz 1845: 6-7 con nota 16; Sekunda 2014: 135. 
ste calzature fa riferimento anche il patriarca bizantino Fozio (IX sec.) nel riassunto della Vita del filosofo Isidore di Damascio di Damasco (cod. 242, 89: 'Y

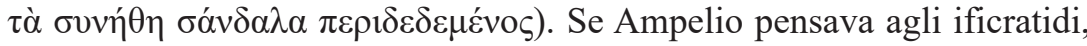
probabilmente doveva usare fonti diverse da Nepote, che tace su questo argomento.

La descrizione in Liber memorialis è molto concisa, ma dobbiamo ricordare che l'unico noto autore greco, che presenta la riforma di Ificrate in modo più ampio, è il summenzionato Diodoro Siculo, la cui narrativa (XV 44) tra l'altro assomiglia anche alla versione di Nepote. D'altra parte, Senofonte, un autore contemporaneo a Ificrate ed interessato ai militari, tace completamente della sua riforma. Evidentemente le innovazioni dell'Ateniese suscitarono invece la curiosità dei Romani. Nella letteratura latina, molta più attenzione è dedicata ad esse, che alle riforme di Filippo II. Se accettiamo l'ipotesi, che il sovrano macedone abbia usato le idee di Ificrate (suo fratello adottivo), questa situazione non sarà sorprendente. Inoltre, si armonizzarebbe con le citate osservazioni degli autori romani sul rapporto familiare tra Ificrate e gli Argeadi. Per i Romani poteva anche essere importante sottolineare l'importanza e le conseguenze dell'innovazione negli armamenti.

La soprammenzionata supposizione sembra essere rafforzata dallo stesso Ampelio, che un po' più avanti riferisce delle innovazioni militari introdotte da $\mathrm{Cabria}^{27}$, un collega di Ificrate, che ha insegnato ai soldati "l'arte dei gladiatori" (Amp. 15, 18: Chabrias dux, qui gladiatoria arte pugnare militem docuit). Questa affermazione è molto problematica a causa della mancanza ad Atene di qualsiasi analogia con il fenomeno romano dei combattimenti tra gladiatori. È possibile che Ampelio abbia frainteso in qualche modo le parole di Cornelio Nepote sulla manovra di opliti di Cabria in Beozia, che, di fronte all'imminente esercito spartano (nel 378) adottarono un atteggiamento con scudi basati su ginocchia e lance rivolte in avanti (Chab. 1, 2: namque in eo victoria fidentem summum ducem Agesilaum, fugatis iam ab eo conducticiis catervis, eo frustratus est, quod reliquam phalangem loco vetuit cedere obnixoque

27 Su Cabria vedi Parke 1933: 56-57, 73-74; Pritchett 1974: 72-77; Bettali 2013: 93-98, 224-228; Duszyński 2016: 71-82. 
genu scuto, proiecta hasta impetum excipere hostium docuit. $)^{28}$. Quindi, il re Agesilao, comandante degli avversari, fermò l'attacco. Naturalmente questo solleva l'altra questione, di come Ampelio abbia associato l'intera situazione ai gladiatori. Nel suo manuale si possono trovare vari cataloghi, mnemonici e semplificazioni, che aiutano a comprendere e ricordare il materiale ${ }^{29}$. Va ricordato che l'intera opera era probabilmente indirizzata a un giovane, forse un ragazzo, quindi, si può presumere, che il riferimento ai gladiatori fosse finalizzato ad adattare il contenuto del capitolo al livello dello studente romano introducendo concetti a lui familiari. Allo stesso modo altrove i tirannicidi ateniesi Armodio e Aristogitone vengono definiti da Ampelio come "i plebei" $(15,8)$. Combattere "alla maniera dei gladiatori" potrebbe significare semplicemente un combattimento spettacolare, sorprendente e basato sui trucchi ${ }^{30}$. Può darsi che, in questo modo, l'autore abbia voluto anche fare riferimento a un migliore allenamento di scherma degli opliti di Cabria.

D'altra parte non si può escludere che Cabria abbia partecipato alle riforme apportate da Ificrate e la relazione di Ampelio si riferisce alle modifiche all'equipaggiamento della fanteria. È noto che nel 369 Cabria comandò un esercito inviato nel Peloponneso in aiuto di Sparta alle prese con i Beoti. Un anno prima, Ificrate aveva condotto una spedizione simile. Inoltre, Plutarco afferma che un alleato di Atene, Epaminonda ricevette un rapporto sull'invio nel Peloponneso di un esercito ateniese dotato di nuovo equipaggio (Mor. 193f). Forse, quindi, Cabria partecipò agli esperimenti condotti da Ificrate e nel testo di Ampelio troviamo il riflesso distorto di questi avvenimenti.

La figura di Ificrate appare anche due volte negli esercizi retorici latini. Il primo caso riguarda le Controversiae di Lucio Anneo Seneca il Vecchio (c. 54 a.C. - 40 d.C.), in cui è stato presentato il presunto processo del comandante dopo la sua spedizione senza successo in Tracia,

28 Una descrizione simile dell'incidente è presentata da Diodoro Siculo (XV 32-33) e Polieno (II 1, 2), secondo i quali le lance degli opliti di Cabria erano puntate verso l'alto e non spostate in avanti. La versione degli autori greci sembra essere più comprensibile, perché in questo caso si tratta probabilmente della solita posizione di "riposo", la cui adozione di fronte agli avversari poteva dimostrare autocontrollo e disciplina dell'esercito ateniese. Vedi Anderson 1963.

29 Cfr. Kołoczek 2016b: 54; 2019: 75-76; vedi anche Sage 1978: 221.

30 Vedi Kołoczek 2019: 73. 
dove fu inviato contro l'anonimo sovrano. Ificrate, che perse due volte la battaglia, fu accusato di tradimento: di aver stringere un'alleanza con il re di Tracia, confermata dal matrimonio con sua figlia (Controv. VI 5: Missus Iphicrates adversus Thracum regem, bis acie victus foedus cum eo percussit et filiam eius uxorem duxit). Quando il destino di Ificrate era in bilico davanti al tribunale, estrasse la propria spada. Allo stesso tempo apparvero alcune persone armate alla maniera della Tracia (quidam Thracum cultu armati). Di fronte a tale dimostrazione i giudici votarono per l'assoluzione completa dell'imputato. Quindi, tuttavia, il capo fu accusato di usare la forza durante il processo. Difendendosi rispose che aveva estratto un'arma per suicidarsi in caso di giudizio avverso. Una breve descrizione della stessa situazione può essere trovata anche nella serie di declamazioni attribuite a Marco Fabio Quintiliano (c. 35 - c. 96). Nella sua versione (Orat. 386) l'imputato Ificrate venne con una spada visibile e portò in tribunale il sovrano tracio Cotys (secondo Plutarco suo suocero). Tuttavia Quintiliano non menziona gli altri difensori del comandante, tanto più i suoi sostenitori armati.

Entrambe le versioni dell'episodio sembrano essere vagamente basate su eventi autentici. È possibile che Ificrate avesse trascorso molto tempo in Tracia e vi avesse sposato una principessa della famiglia di Seute II, probabilmente, come riferisce Demostene (XXIII 219), la sorella di Cotys ${ }^{31}$. Tuttavia, non ci sono prove che in Tracia il comandante

31 Vedi anche Athen. IV 131a-f, che cita un ampio estratto della commedia Protesilao del poeta contemporaneo di Ificrate, Anassandride, in cui fu deriso il matrimonio del capo ateniese. Il problema della relazione di affinità tra Ificrate e Cotys è complesso. Demostene (la nostra prima fonte) per descriverla usa la parola $\kappa \eta \delta \varepsilon \sigma \tau \eta \dot{s}$, che può

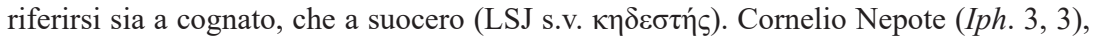
però, descrive la consorte tracia di Ificrate come Coti regis filia. Allo stesso modo Ate-

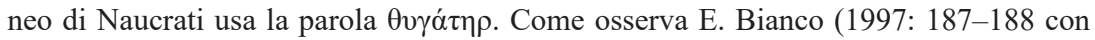
la nota 29), Menesteo, nato da questa unione, ricoprì l'incarico di stratega nel 357/6 a.C. A causa del limite dell'età (30 anni) dovette nascere al più tardi negli anni ' 80 del IV secolo. Pertanto, la moglie di Ificrate doveva essere stata abbastanza matura per sposarsi in quel momento. Nel frattempo, secondo Demostene (nel discorso Contro Aristocrate XXIII 163), Cotys dopo la sua morte intorno al 360 a.C. lasciò i figli minori. È improbabile, che ci sarebbe stata una differenza di età altrettanto grande (oltre 20 anni) tra la prole del sovrano. Pertanto, sembra più plausibile, che Ificrate abbia sposato non figlia, ma la sorella di Cotys, sebbene questo problema sia difficile da risolvere senza ambiguità. 
avesse ricoperto la carica di stratega ateniese. Gli studiosi contemporanei concordano sul fatto che vi era come mercenario ${ }^{32}$. Non sappiamo nulla delle possibili sconfitte subite da Ificrate in questa regione. Gli altri autori antichi suggeriscono il contrario. Polieno presenta una serie di strategemmi di successo contro i Traci (III 9, 4; 41; 46; 60; 62). Nepote, d'altro canto, menziona il ripristino del potere di Seute II da parte dell'Ateniese (Iph. 2, 1).

Il processo di Ificrate presentato da Seneca e Quintiliano probabilmente ebbe luogo, ma in circostanze completamente diverse, ossia alla fine (o dopo la fine) della guerra di Atene con ex alleati della seconda lega delio-attica ${ }^{33}$. Apparentemente Quintiliano si riferisce a questa faccenda nell'Institutio oratoria, forse basando sul racconto di Aristotele di uno scambio di opinioni tra Ificrate e l'oratore Aristofonte che lo accusò di tradimento (Quint. Inst. orat. V 12, 10; cfr. Arist. Rhet. II 23, 7). Va notato che, mentre nella versione di Aristotele la mazzetta avrebbe dovuto

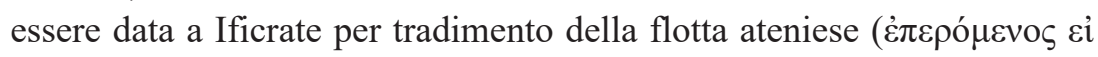

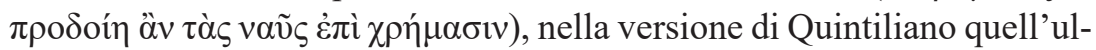
timo argomento era completamente omesso. Tutto l'aneddoto è stato accostato a un episodio analogo della storia romana: Marco Emilio Scauro, un senatore ampiamente rispettato fu accusato - durante la guerra sociale - contro gli alleati italici (nell'anno 90 a.C.) - del tradimento della repubblica romana dal tribuno della plebe Quinto Vario Severo, chiamato l'Ibrida, a causa della sua origine mista. Scauro si difese, sottolineando che, come l'ex censore e console, aveva indubbiamente meno motivi per tradire lo stato dello stesso accusatore (Ibrida fu il primo senatore romano proveniente dall'estero, più precisamente dalla Spagna). Sappiamo da altre fonti che l'accusa si basava sul sospetto della presunta accettazione da Scauro di una mazzetta dal re Mitridate VI del Ponto, che sosteneva gli insorti italici (vedi Val. Max. III 7,8). Il contesto di questo aneddoto e la sua giustapposizione con la relazione del processo di Ificrate, che si difendeva in modo molto simile, sembra indicare che Quintiliano, come negli sopramenzionati esercizi retorici, anche se si basava sul racconto

32 Parke 1933: 55-56; Pritchett 1974: 64-66; Bianco 1997: 186-188; Bettali 2013: 374-376; Sears 2013: 125-127.

33 Sulla guerra sociale vedi Accame 1941: 189-195; Cawkwell 1962: 34-40; Cargill 1981: 171-182; Parker 1986: 22-34. 
di Aristotele, poteva ancora una volta avere in mente l'alleanza tra il comandante ateniese e il sovrano tracio Cotys.

Intanto, come già accennato e come si può presumere da altre fonti, l'accusa di Ificrate da parte di Aristofonte riguardava la battaglia navale di Embata che ebbe luogo molto più tardi (nel 356) nello stretto tra Chio e l'Asia Minore. In quel tempo, a causa del maltempo, Ificrate rifiutò di partecipare all'attacco alla flotta di avversari (le forze di Chio, Rodi e Bisanzio), nonostante ciò, un altro comandante ateniese, Carete, decise di combattere da solo e subì gravi perdite a causa della tempesta (vedi Diod. XVI 21) ${ }^{34}$. In un atto di vendetta Carete, in seguito, accusò (in collaborazione con Aristofonte) Ificrate e altri strateghi (Menesteo, il figlio di Ificrate, e Timoteo) di tradimento. Il processo si concluse con l'assoluzione degli imputati, ad eccezione di Timoteo, condannato a un'alta multa ${ }^{35}$. Il re Cotys non poteva parteciparvi, perché era già morto da diversi anni ${ }^{36}$. Tuttavia, nel contesto di questo caso si registra il motivo di intimidazione alla corte ateniese da parte di Ificrate, e ciò consente di identificare l'intero episodio con il processo descritto negli esercizi retorici di Seneca e Quintiliano. Polieno descrive due situazioni, in cui Ificrate davanti al tribunale riuscì efficacemente a intimidirlo. A causa del contenuto molto simile di entrambe le descrizioni, probabilmente stiamo trattando qui il cosiddetto doppione, cioè descrizione di un solo evento come due episodi separati ${ }^{37}$. Nel primo strategemma Ificrate circondò il tribunale con giovani uomini

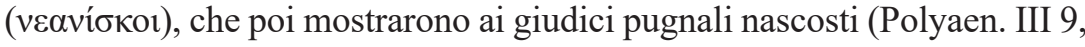
15). Nella seconda variante il comandante mostrò la propria spada, suggerendo che tutta l'area era circondata da membri della sua eteria (Polyaen. III 9, 29). Il corso dell'incidente in entrambi i casi è quindi simile alla versione latina. Tuttavia Polieno tace sulla reazione più decisiva del tribunale

\footnotetext{
$34 \quad$ Vedi Pritchett 1974: 72; Parker 1986: 29-31.

35 Hansen 1975: 101.

36 Archibald 1998: 221.

37 M. Borowska (2003: 127) nel suo commento all'edizione polacca di Polieno suggerisce che il primo degli incidenti descritti da lui (III 9, 15) potrebbe anche riferirsi al processo di erezione della statua di Ificrate ad Atene. Tuttavia contro questa opzione va notato che l'antico autore parla della minaccia del comandante con la pena di morte. Ificrate invece, secondo Nepote $(\operatorname{Iph.3,3)}$ si trovò in una situazione così grave solo una volta, durante la guerra sociale.
} 
al tentativo di intimidazione di Ificrate, menzionando solo i commenti non ufficiali sull'incidente (III 9, 29).

Naturalmente l'intera situazione potrebbe essere sorprendente. Questo è forse l'unico caso della letteratura antica dove leggiamo dell' intimidazione della corte di Atene. Allo stesso tempo ci sono però una serie di motivi per cui la relazione di Polieno non può essere rifiutata. Innanzitutto l'autore della raccolta di stratagemmi era probabilmente ben informato sul processo: indica l'esatto luogo di confronto durante la guerra sociale (Embata) e nomina due accusatori degli strateghi - non solo Carete, ma anche Aristofonte, la cui partecipazione al processo è confermata da Dinarco (Din. I 14) e Claudio Eliano (Var. hist. XIV 3) ${ }^{38}$. In secondo luogo, Polieno verosimilmente ha preso le sue informazioni sull'intimidazione del tribunale da parte di Ificrate da due fonti separate, non notando che si riferivano allo stesso caso $^{39}$. Altrimenti è difficile spiegare il fatto di un doppione nella sua opera. Pertanto un numero maggiore di autori antichi avrebbe dovuto evocare questo aneddoto, che si riflette anche nella citata letteratura latina. L'esistenza di ben quattro descrizioni dell'incidente nelle opere di tre diversi autori suggerisce che la tradizione dell'eccezionale comportamento di Ificrate fosse piuttosto ricca. Quindi, è possibile che, quando apparve in tribunale negli anni ' 50 , il famoso stratega ateniese effettivamente inventò siffatto stratagemma (in generale, non nei dettagli).

Oltre alla credibilità dello stesso aneddoto, i dubbi possono essere causati dalla menzione di Polieno sul possesso da parte di Ificrate di un gruppo di sostenitori ad Atene (eteria) ${ }^{40}$. Altre fonti non lo menzionano, così come il funzionamento di tali associazioni nel IV secolo a.C. Gli studiosi hanno formulato diversi suggerimenti per risolvere questo problema. J. Roberts ritiene che al processo fosse apparso un gran numero di soldati di Ificrate, legati al loro comandante ${ }^{41}$. N. Sekunda, a sua volta, è

38 Sul processo degli strateghi vedi Cawkwell 1962: 45-49; Hansen 1975: 100; Bianco 1997: 203-204. Eliano non fa menzione di Ificrate, ma si riferisce all'accusa di Timoteo, che stesso si astenne dall'attacco presso Embata. Entrambi i comandanti furono processati insieme.

$39 \quad$ Pecorella Longo 1971: 65.

40 Sul problema del funzionamento dei gruppi politici ad Atene nel IV secolo a.C. e la loro classificazione come eterie vedi Pecorella Longo 1971: 64-66.

41 Roberts 1982: 49. 
dell'opinione che quelli fossero i giovani frequentatori dei ginnasi reclutati dai fedeli lochagoi (i veterani delle precedenti campagne di Ificrate) ${ }^{42}$. Naturalmente l'intera situazione avrebbe potuto essere un bluff da parte del comandante, consistente nel creare tra i giudici una convinzione del presunto pericolo. Ciò è indirettamente indicato dalla versione di Quintiliano e dal secondo dei stratagemmi di Polieno, in cui gli armati sostenitori di Ificrate non appaiono fisicamente. Anche così, il trucco per essere efficace doveva essere considerato probabile. Ciò significa che il comportamento di Ificrate fu valutato dai giudici come una vera minaccia da non sottovalutare.

Negli esercizi retorici latini vediamo un'evidente confusione di vari episodi della vita del comandante (la sua attività in Tracia come mercenario e durante la guerra sociale come stratega ateniese $)^{43}$. Ciò non sorprende, tuttavia, perché la coerenza delle situazioni descritte con i fatti storici non era cosa particolarmente importante in questo tipo della letteratura ${ }^{44}$. Va anche notato che la visione del famoso stratega ateniese, che entrava in alleanza con i sovrani traci, cercava di intimidire la corte, e per di più minacciava di suicidarsi, senza dubbio attirava l'attenzione, commuoveva, forniva anche intrattenimento e, di conseguenza, era un ottimo esempio da utilizzare sia nelle scuole retoriche, che nelle declamazioni artistiche, dominanti durante il periodo dell'Impero. Dopotutto gli altri esempi di controversiae fatte da Seneca solo nel VI libro riguardano argomenti sensazionali come avvelenamento, tradimento di una vestale o il processo di un padre che ha ceduto sua moglie a uno dei suoi figli cercando di salvarlo dalla malattia ed è stato accusato dall'altro di follia. A Quintiliano a sua volta tra l'altro dobbiamo la descrizione del processo, durante il quale l'oratore Iperide svelò davanti ai giudici ateniesi il corpo nudo dell'etera Frine, da lui difesa (Inst. orat. II 15, 7-9). La figura di Ificrate nelle opere di Seneca e Quintiliano era quindi probabilmente solo una maschera storica volta ad autenticare, o piuttosto a concretizzare, l'esempio retorico. Il semplice uso della sua figura in questo tipo di raccolta retorica dimostra che si trattava di una persona ben nota ai Romani dell'e-

\footnotetext{
$42 \quad$ Sekunda 2019: 85.

43 H. Parke (1933: 56), commentando la relazione di Seneca, afferma: it is so garbled as to be valueless. Per quanto riguarda il fenomeno della contaminazione di diversi episodi dagli autori latini vedi Appel 2011: 17, 23-25.

$44 \quad$ Vedi Kołoczek 2019: 82-89.
} 
poca, poiché altrimenti le controversie e gli esempi basati sulla sua storia sarebbero state poco comprensibili e, di conseguenza, inutili per i lettori.

Particolarmente intrigante è il motivo di Ificrate che suggerisce la volontà di suicidarsi con una spada se fosse stato condannato. Naturalmente, secondo gli autori degli esercizi retorici questa era una scusa per proteggerlo dall'accusa di intimidazione della corte. L'intera situazione, tuttavia, è abbastanza simile all'aneddoto associato ad un altro stratega, Pachete, che, ritenuto colpevole, apparentemente si tolse la vita. Il problema è che le circostanze di questo ultimo caso a noi sono ignote e tutto l'episodio lo conosciamo solo dalle opere di Plutarco, un autore moralista, inoltre, vivente 500 anni dopo Pachete (Plut. Arist. 26, 3; Nic. 6, 1). Tucidide, invece (interessato alle azioni legali contro gli strateghi che lui sperimentò personalmente), tace su questo argomento. Per quanto riguarda lo stesso fenomeno del suicidio onorario, ne conosciamo solo alcuni esempi nel mondo greco dell'età classica e tutti riguardano i comandanti che hanno fallito ${ }^{45}$. Pertanto, come indica J. Roberts, è necessario prestare qualche cautela in relazione al riferimento di Plutarco ${ }^{46}$.

Ificrate sembra essere una figura piuttosto ben nota ai Romani dei primi due secoli dell'impero, poiché senza una parola di introduzione appare nelle opere popolari degli autori come Seneca il Vecchio, Quintiliano ed Ampelio. Neanche Nepote si sentiva obbligato a giustificare la presenza del comandante ateniese nella sua collezione. Questo è un interessante contrasto con il caso del famoso Pelopida, la cui biografia inizia con l'affermazione di Plutarco che questo comandante è più familiare agli storici che ai lettori ordinari (Pel. 1, 1: Pelopidas Thebanus, magis historicis quam vulgo notus). Per i Romani Ificrate rimase soprattutto un maestro nell'arte militare, ma loro facevano anche i riferimenti al suo talento nella retorica. Inoltre si può notare che gli elementi enfatizzati dagli autori latini (legami con gli Argeadi, innovazioni degli armamenti, giovane età dell'inizio della sua carriera, passione per la disciplina) sono moderatamente esposti nella tradizione greca. D'altra parte venivano

45 W.K. Pritchett (1974: 32) elenca Timocrate di Sparta (in seguito alla battaglia navale di Naupatto del 430 - Thuc. II 92, 3) Crinippo di Siracusa (catturato da Ificrate a Corcira nel 372 - Xen. Hell. VI 2, 36) ed Andromaco di Elea (dopo l'invasione degli Arcadi all'Elide del 365/4 - Xen. Hell. VII 4, 19).

46 Roberts 1982: 140. 
comunemente riconosciute le competenze dell'Ateniese nell'invenzione di stratagemmi, non solo sul campo di battaglia, ma anche in tribunale.

Il ricordo di Ificrate svanì quasi completamente nelle epoche successive. Il motivo principale di questa situazione può essere considerato la perdita di molte fonti, che probabilmente parlavano di più del comandante ateniese (come per esempio Eforo, Teopompo o Pompeo Trogo). Nel materiale disponibile, le informazioni su Ificrate sono disperse. Un fattore importante era anche il corso della sua carriera senza grandi vittorie o conquiste. Nonostante ciò la figura del comandante apparve in diversi passi interessanti della letteratura dell'epoca moderna.

Il capo ateniese appare in una delle opere di Michele Marullo Tarcaniota (Costantinopoli 1453 - Volterra 1500), un soldato e poeta greco che, residente in Italia, scriveva in latino. La poesia di Marullo, fortemente radicata nella cultura greca, intrisa di nostalgia per la patria perduta e orientata sul passato, era allo stesso tempo un tipico prodotto della cultura umanistica italiana. Nelle Hymni naturales, imitando gli inni orfici, il poeta romano Lucrezio e simultaneamente attingendo alla tradizione del neoplatonismo fiorentino, Marullo elogiava il potere divino della natura. A sua volta, nei quattro libri degli epigrammi dedicati a Lorenzo di Pierfrancesco de' Medici (1463-1503), cugino di Lorenzo il Magnifico, che allora governava Firenze, il poeta esule greco riferiva a tutti questi argomenti, che occupavano in quei tempi l'élite intellettuale: l'amore, il sentimento verso l'antichità, le discussioni estetiche e filosofiche contemporanee. Inoltre prestava la sua attenzione poetica alla ricerca della propria identità nella nuova realtà ${ }^{47}$.

Questo vale anche per il suo epigramma su uno dei figli di Ificrate, Menesteo:

Matrem rogatus faceret an patrem pluris

Iphicrate satus nobili,

"Matrem" inquit: "haec enim Atticum edidit, Graecus

Quem voluerat Thracem pater."

(Epigr. I 56 de Mnestheo in: Michaelis Marulli Carmina, ed. A. Perosa, Zürich 1951)

$47 \quad \mathrm{Su}$ Michele Marullo e la sua poesia vedi Copponi 2008. 
Marullo, originario della Grecia, scrivendo sullo stratega ateniese avrebbe potuto fare un riferimento ad una delle numerose opere scritte nella sua lingua madre, ma decise di fare riferimento alla breve biografia scritta da Cornelio Nepote (cfr. Iph. 3: Menesthea filium reliquit ex Thraessa natum, Coti regis filia. Is cum interrogaretur, utrum pluris, patrem matremne, faceret, 'Matrem'inquit. Id cum omnibus mirum videretur, 'At' ille 'merito' inquit 'facio. Nam pater, quantum in se fuit, Thraecem me genuit, contra ea mater Atheniensem). In questo modo, il poeta incontrò senza dubbio le aspettative della gente della sua epoca. Nel Medioevo l'interesse per le opere di Nepote è difficile da notare, invece nel Cinquecento si può osservare un rapido aumento della sua popolarità, la cui prova migliore è rappresentata dai 100 manoscritti conservati di questo secolo ${ }^{48}$. Apparentemente il testo di Marullo è solo una concisa parafrasi dell'episodio raccontato da Nepote. Però la scelta dello stratega ateniese certamente non era casuale. La risposta paradossale del figlio di Ificrate, che sottomette al suo famoso padre la sua anonima madre tracia, che voleva che lui appartenesse alla cultura ateniese, come notò M. Jansen, sembra corrispondere bene alla situazione dello stesso Marullo. Questo soldato e rifugiato dallo stato ottomano, anche dopo aver trovato una nuova patria, sottolinea il suo rapporto con la cultura greca lasciata alle spalle. Si richiama l'attenzione speciale sul suo spostamento dell'attenzione dalla persona del famoso padre alle sue origini (nella versione di Nepote era piuttosto quello, che Menesteo doveva al padre il suo luogo di nascita e alla madre l'educazione greca). Più tardi Menesteo, come suo padre, divenne uno stratega, il che significa che senza dubbio era cittadino ateniese a pieno diritto. Ciò è interessante, perché probabilmente lo stesso Marullo non proveniva da Costantinopoli, ma si sentiva molto legato a questa città, così come testimoniato dalla sue poesie ${ }^{49}$. Tuttavia, l'osservazione di Jansen può essere ulteriormente sviluppata. Il figlio di Ificrate doveva il suo nome al leggendario re di Atene, Menesteo, un partecipante alla guerra di Troia, che ci dice molto sull'auto-presentazione e l'autostima del comandante ateniese, collegato, come ricordiamo, con la famiglia reale della Macedonia $^{50}$. Non si deve dimenticare che, ai tempi di Marullo, l'associazione con

48 Sulla tradizione manoscritta delle opere di Cornelio Nepote vedi Marschall 1977.

49 Jansen 2009: 227-229.

50 Vedi Sears 2013: 65. 
la guerra di Troia poteva riferirsi anche alla Turchia ottomana e al recente crollo dell'impero bizantino, che costrinse i suoi abitanti greci a rivedere il loro atteggiamento nei confronti del vecchio nemico. In questo contesto la scelta fatta da Menesteo diventa non solo una scelta consapevole e generazionale della propria identità, ma anche una verifica delle decisioni prese una volta dai suoi genitori.

La questione del comportamento "tracio" o meglio "rustico" dello stesso Ificrate è presente non solo nell'opera di Nepote, ma anche nelle fonti greche. Polieno descrive una situazione in cui il cognato di Ificrate gli chiese sorpreso perché fosse sempre in armatura. Il comandante rispose che pratica la vigilanza (Polyaen. III 9, 16). La chiave per l'interpretazione di questo incidente sembrano le parole di Tucidide secondo il quale gli ateniesi sarebbero stati i primi tra i greci, ad abbandonare l'abitudine di camminare quotidianamente con le armi (I 6, 1-4). Nel testo di Polieno ci stiamo quindi occupando dell'inversione completa dei comportamenti identificati con i barbari e con gli Elleni. Ificrate rimane in costante disponibilità alla guerra, che sorprende persino suo cognato, re dei Traci Odrisi, e quindi un barbaro par excellence. Di conseguenza l'intero episodio assume un carattere paradossale e comico. Nel contesto della situazione descritta è notevole anche la testimonianza di Dionigi di Alicarnasso su Ificrate come oratore (Lys. 12). Lo storico greco con scetticismo fa riferimento alla teoria, secondo la quale il famoso logografo Lisia sarebbe stato il vero autore dei testi delle orazioni attribuite al comandante. A suo avviso questa opinione non è convincente sia dal un punto di vista cronologico (alcuni di questi discorsi riguardavano le questioni avvenute svolte molti anni dopo la morte di Lisia), che stilistico. Secondo Dionigi lo stile di questi discorsi è caratterizzato non tanto dall'ingegnosità retorica quanto dall'orgoglio e dalla vanteria di un

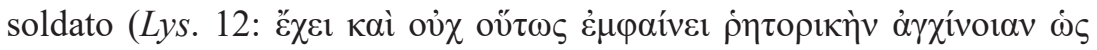

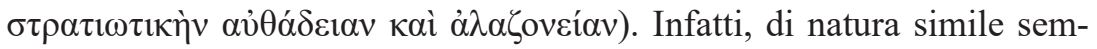
brano essere alcuni dei frammenti conservati delle presunte orazioni. Per esempio in uno di essi leggiamo che Ificrate una volta avrebbe detto agli Ateniesi: "Se solo aveste sette strateghi come me, Lacedemone sarebbe

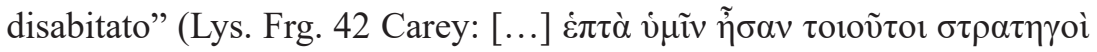

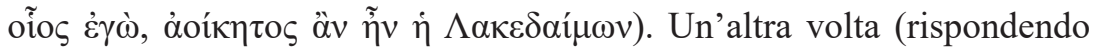
ad un certo Armodio, discendente del famoso tirannicida, che derideva le sue umili origini), avrebbe detto che la sua casa iniziava con lui stesso 
(Lys. frg. 45) ${ }^{51}$. Forse, quindi, fu Ificrate a essere uno dei prototipi del carattere di Soldato Glorioso, conosciuto dalla commedia attica nuova e dalla palliata romana. Questa è solo un'ipotesi, perché tutti gli esempi di tali personaggi a noi noti provengono da tempi chiaramente successivi e nessuno di loro si riferisce direttamente al capo ateniese. Tuttavia, la figura di Ificrate sembra corrispondere bene ad alcune delle caratteristiche di questo personaggio, in particolare la sua tendenza a mostrare ed esagerare i propri successi. Significativa in questo contesto è anche la

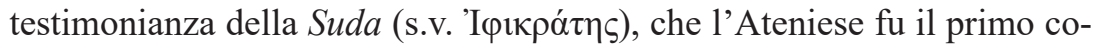

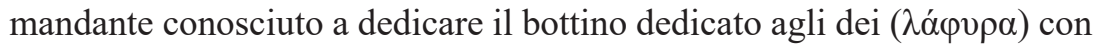
il proprio nome, perché in precedenza su di esso veniva collocato solo il nome della polis vittoriosa.

Un altro riferimento alla figura di Ificrate proviene anche dall'Italia e riguarda le sue attività militari. Niccolò Machiavelli nel suo trattato Dell'arte della guerra, pubblicato nel 1521 si riferisce sinteticamente alla situazione, in cui Ificrate "ammazzò una guardia che dormiva, dicendo di averlo lasciato come l'aveva trovato". Questo brevissimo aneddoto, insieme ad altri antichi esempi citati dall'autore, stava a dimostrare, quanto fosse importante la disciplina in una città minacciata da un attacco dall'esterno (Dell'arte della guerra VII, p. 360 ed. A. Capata, Roma 2011). Tutta la descrizione è stata senza dubbio presa dall'opera di Frontino (Strat. III 12, 2), l'autore regolarmente citato nella letteratura del primo periodo moderno.

Frontino era un autore ben noto anche in Polonia ${ }^{52}$. Stanislao Sarnicki (1532-1597), uno storico, attivista e polemista calvinista e anche un autore ed editore di opere legate alla guerra, usò gli stratagemmi del

51 I riferimenti alla derisione delle umili origini di Ificrate corrispondono bene alle

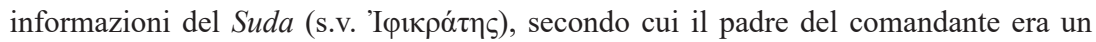
semplice calzolaio. Pausania (I 24, 7) ci ha dato anche il suo nome - Timoteo, ma questo è tutto ciò che sappiamo di lui. Il padre del comandante non è menzionato in alcun modo come una persona impegnata pubblicamente, che compie liturgie, ecc. In effetti la tradizione della modesta origine di Ificrate sembra essere credibile.

52 La questione della ricezione dell'opera di Frontino nelle terre polacche discute B. Burliga (2016: 23 con nota 5). L'autore romano è citato per esempio da Giacobbe Przyłuski nel VI libro dei suoi Leges seu statuta ac privilegia Regni Poloniae (1553), Alberto di Hohenzollern nel Kriegsordnung (l'opera pubblicata nel 1555, tradotta in polacco nel 1561), e da Martino Bielski nel trattato Sprawa rycerska ("L'arte cavalleresca”), pubblicato nel 1569 . 
Frontino nei suoi Libri Etmani (in polacco Księgi Hetmańskie, pubblicati intorno al $1577^{53}$ ), presentando la stessa situazione che Machiavelli descrisse un mezzo secolo prima (vedi p. 274, 278 ed. M. Ferenc, Cracovia 2015). Nel suo lavoro Sarnicki menziona Frontino più volte (p. 4, 12, 51 ), ciò indica che anche quell'aneddoto è stato preso direttamente da lui. Molto probabilmente l'autore polacco ha usato Scriptores rei militaris, un'edizione collettiva degli antichi scrittori militari (Frontino, Eliano Tattico, Vegezio e Modesto), a cura di Filippo Beroaldo (Philippus Beroaldus) o di Giovanni Sulpizio da Veroli (Johannes Sulpitius Verulanus) ${ }^{54}$. J. Sikorski osserva anche un interessante parallelo tra la composizione dell'opera di Machiavelli e quella di Sarnicki. Le questioni sollevate dall'autore polacco (attività legate alla preparazione alla guerra, condotte durante e dopo il suo completamento) sono presentate nello stesso ordine del libro IV del trattato di Machiavelli ${ }^{55}$. Forse Sarnicki si inspirò all'opera del famoso Fiorentino, sebbene da nessuna parte si riferisca direttamente a lui.

La figura di Ificrate non era estranea neanche a Giacobbe Przyłuski, l'autore di Leges seu statuta ac privilegia Regni Poloniae. Nel sesto libro della sua opera monumentale (pubblicata nel 1553, quindi prima dell'opera di Sarnicki), dedicata agli affari militari, l'autore espone alcuni esempi di trucchi usati dagli antichi comandanti ${ }^{56}$. Tra questi, sei riguardano Ificrate (pp. 928-930). Si pensava che Przyłuski riscrivesse semplicemente Frontino ${ }^{57}$. Tuttavia alcuni di questi episodi non compaiono affatto nell'opera dell'autore romano, ma li troviamo nel lavoro di Polieno (Polyaen. III 9, 8; 13; 22; 23; 32). Ciò suggerisce un uso diretto della collezione greca di stratagemmi dall'autore polacco. È difficile però definire, quale edizione abbia usato. I casi da lui selezionati riguardano varie questioni: modi per sollevare il morale, circostanze in cui si dovrebbe (o no) combattere; cooperazione di varie formazioni sul campo di battaglia o addestramento dei soldati.

\footnotetext{
53 Sulla data della redazione dell'opera di Sarnicki vedi Sikorski 1967:15-16; Ferenc 2015: XIV.

54 Sikorski 1967: 36; Lichański 2009: 107. Per le ulteriori informazioni sulle moderne edizioni di Frontino vedi Burliga 2016: 22 con nota 3.

55 Sikorski 1967: 49.

56 Sull'opera di Przyłuski vedi Sikorski 1966: 65-67.

57 Sikorski 1966: 66.
} 
Un altro insolito esempio di descrizione della figura del comandante ateniese lo incontriamo nella commedia in un atto di Pierre de Marivaux (1725) L'isola degli schiavi (L'Île des esclaves). La trama è molto semplice. In seguito ad un naufragio quattro sopravvissuti: Iphicrate, Euphrosine e i loro servi Cléanthis ed Arlequin si trovano su un'isola misteriosa governata da schiavi fuggiti. Il sovrano dell'isola, Trivelin, costringe i naufraghi a scambiare i loro ruoli, il che dopo una serie di colpi di scena porta a sanare i rapporti tra gli eroi e alla loro liberazione dai costumi a loro imposti dalla società. Siamo particolarmente interessati alla figura di Iphicrate prima della sua trasformazione, specialmente perché l'ispirazione al comandante ateniese del IV secolo a.C. sembra chiara. Iphicrate di Marivaux è un uomo arrogante, severo e imperioso, a cui piace usare brevi esclamazioni emotive (Hélas! Eh! Comment donc! Misérable! Esclave insolent!). La sua prima frase che nello stesso tempo apre l'intera commedia è un grido per il suo schiavo (“Arlequin!”). Tuttavia, l'elemento più caratteristico del linguaggio del protagonista sono i comandi, a volte disposti in intere serie (Dis-moi!”, „Ne perdons point de temps!”; „suis-moi: ne négligeons rien pour nous tirer d'ici!" "Parle donc, as-tu perdu l'esprit!?"). Alla fine della prima scena, il linguaggio di Iphicrate viene caratterizzato da Arlequin come la "lingua di Atene" (Ah! Ah! Vous parlez la langue d'Athènes; mauvais jargon que je n'entends plus), in altre parole, la lingua dei signori, anche se a lungo questo termine poteva anche essere un'allusione al dominio ateniese su mare.

È difficile indicare quanto Marivaux nella costruzione del suo protagonista si basasse su fonti antiche o studi successivi, che menzionano i rapporti di Ificrate con i sovrani di Macedonia e Tracia, e quanto fosse guidato da associazioni, per esempio con il suo nome, che in greco significava letteralmente qualcuno che governa (regna) mostrando la sua

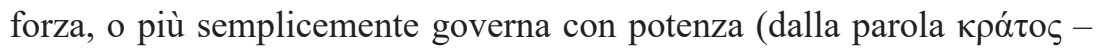
"forza, potenza" e l'avverbio $\tilde{i} \varphi \mathrm{l}$ - "con forza" o "con potenza"). Nei testi omerici l'avverbio î $\varphi$ ı è combinato con gli altri verbi relativi alla

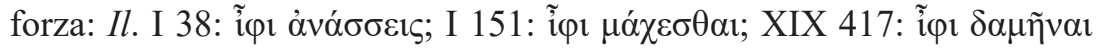
$=O d$. XVIII 156; mantenendo la grafia invariata appare anche in testi di autori successivi, ad esempio Euforione di Calcide, un poeta e grammatico del III secolo a.C. (fr. 90); può anche essere visto nel nome della mitica Ifigenia ("nata con potere") - originariamente uno degli epiteti di culto di Afrodite, e persino, come assumono gli autori di lemma nel 
lessico di Liddell e Scott, si appare su una delle tavolette di Micene con l'iscrizione wi-pi-no-io ${ }^{58}$. Lo stesso nome del comandante, si deve considerare molto raro (conosciamo solo pochi dei suoi titolari, incluso il figlio dello stratega: Ificrate il Giovane ${ }^{59}$ ); certamente in qualche modo lo nobilitava e poteva avere una certa influenza sulla sua percezione come l'uomo forte e determinato. Anche se Marivaux si basava su fonti che menzionavano l'antico capo, non era interessato alla sua carriera militare e ai suoi successi come stratega o condottiere, ma ancora una volta a ciò, che attirò l'attenzione di Michele Marullo e degli antichi autori romani: il famoso nome del comandante, invariabilmente associato all' antica gloria di Atene e allo stesso tempo ad una certa apertura e cosmopolitismo (dopo tutto, incontriamo il protagonista di Marivaux su un'isola lontana e l'intera commedia termina con la sua riconciliazione con lo schiavo Arlequin).

Per riassumere, sembra, che il ritratto di Ificrate nella letteratura latina sia simile, ma non identico a quello della letteratura greca. Ovviamente, in entrambi i casi è raffigurato come un comandante di talento, eccellente soprattutto in campo di stratagemmi. Tuttavia, si può vedere, che i Romani prestavano più attenzione dei Greci ad alcuni elementi della sua biografia. Si trattava principalmente di Ificrate, che iniziò la sua carriera in giovane età, delle sue innovazioni nel campo degli armamenti, la sua passione per la disciplina, i suoi rapporti familiari con gli Argeadi di Macedonia e i sovrani Traci o dei metodi non convenzionali utilizzati nella sua difesa davanti alla corte ateniese. La letteratura greca, sebbene in essa il comandante viene menzionato molto più spesso, ci fornisce un numero notevolmente inferiore o comparabile di riferimenti a questi argomenti.

Il tema della vittoria di Ificrate nella battaglia del Lecheo, nonostante fortemente esposta da fonti greche, appare nelle opere romane solo nella forma di una frase nella Vita di Ificrate scritta da Nepote. Questo è pienamente comprensibile, perché il successo di Lecheo ebbe un significato fondamentale soprattutto per gli Ateniesi (come simbolo del risorgimento del loro stato dopo la sconfitta nella guerra del Peloponneso) e anche per

\section{$58 \quad$ LSJ, s.v. $\tilde{i} \varphi$ e e Suppl.}

59 Come affermato da N. Sekunda (1994: 303), dell'antico mondo greco sappiamo solo sei persone che portano il nome di Ificrate, di cui quattro sono ateniesi. Fatta eccezione per la figura del nostro comandante e di suo figlio, tutti gli altri sono noti solo da menzioni singole. 
gli altri Greci (disfatta dei migliori opliti della Grecia da parte dei peltasti, una sorta di fanteria leggera, quindi una formazione meno prestigiosa). Per i Romani questi fattori indubbiamente non erano particolarmente importanti. Anche la stessa battaglia del Lecheo non era un evento abbastanza grande, significante e, di conseguenza, attraente. Altri aspetti della biografia di Ificrate potrebbero essere sembrati più interessanti. La giovane età di Ificrate quando divenne un comandante indipendente probabilmente attirò l'attenzione dei Romani abituati ai diversi standard associati al piuttosto rigido cursus honorum. Interessanti per loro potrebbero essere anche i collegamenti dell'Ateniese con la famiglia reale macedone, che nelle persone del fratello adottivo di Ificrate, Filippo II e suo figlio Alessandro Magno portò alla creazione di un impero mondiale, diretto predecessore di Roma ${ }^{60}$. In un contesto simile possiamo spiegare le osservazioni dei Romani sulla riforma della fanteria. Se Filippo II si basava davvero sulle idee di Ificrate, l'Ateniese appariva sicuramente come quello, che gli aveva dato (e ovviamente al suo grande successore) uno degli strumenti per conquistare il mondo. In questo modo Ificrate diventerebbe, per così dire, il padre intellettuale della formazione, con la quale i Romani combatterono spesso sui campi di battaglia in Italia, Grecia e Asia. Ovviamente la figura di Ificrate rimase anche una potenziale fonte di ispirazione per i capi romani come autore di numerosi trucchi di guerra. La sua abilità, combinata con i suoi talenti retorici, divenne evidente anche nella sfera delle lotte giudiziarie, che potrebbero assomigliare alle carriere dei grandi politici romani ${ }^{61}$. Quindi, probabilmente, l'interesse per la persona del comandante ateniese aveva non solo una dimensione storica o antiquaria, ma anche una dimensione pratica.

Per quanto riguarda il problema della successiva ricezione della figura di Ificrate, possiamo vedere che, sebbene poco conosciuto, il comandante ateniese non era completamente estraneo agli eruditi dell'Europa moderna. Particolarmente interessanti erano le azioni intraprese dal

60 Su questo argomento vedi per esempio Vell. Pat. I 6, 6; Suet. Aug. 18; Amp. Lib. mem. 10 e 16; Solin. 9, 21.

${ }_{61}$ Vedi per esempio Sprawski 2011: specialmente pp. 298-299, che, analizzando l'immagine molto positiva di Filippo II nell'opere di Cicerone suggerisce, che questo eccezionale oratore ed ambizioso politico romano potesse sentire una sorta di "parentela" con il re macedone, che non doveva la sua posizione tanto alla sua famiglia e alle sue abilità militari, quanto al suo duro lavoro, ai suoi talenti diplomatici e persuasivi. 
comandante per mantenere la disciplina nell'esercito, motivare i soldati e allevare il loro addestramento. La maggior parte dei riferimenti a Ificrate rientra nel fascino rinascimentale per l'antichità classica, che non ometteva anche il campo della teoria militare. Gli eruditi moderni consideravano come valida l'esperienza dei Greci e dei Romani, quindi si riferivano volentieri agli esempi tratti sia dagli antichi trattati militari, che dalla prosa storica ${ }^{62}$. Le informazioni sui successi di Ificrate venivano prese principalmente da Frontino, anche se a volte, come mostra l'esempio di Giacobbe Przyłuski, usavano anche dalle fonti greche. Inoltre la figura del comandante riguardava i riferimenti più "pacifici" nelle opere di Marullo e Marivaux. L'attenzione di questi autori è stata catturata dalla straordinaria personalità dell'Ateniese (e forse anche dal suo nome originale), che emerge dalle notizie sparse nelle fonti antiche, soprattutto quelle latine.

\section{BIBLIOGRAFIA}

Accame S., 1941, La lega ateniese del secolo IV a.C., Roma.

Alonso-Núñez J.M., 1987, 'An Augustan World History: The 'Historiae Philippicae' of Pompeius Trogus', Greece \& Rome 34/1, pp. 56-72, https://doi. org/10.1017/S0017383500027704.

Anderson J.K., 1963, 'The Statue of Chabrias', American Journal of Archaeology 67, pp. 411-413, https://doi.org/10.2307/501624.

Anderson J.K., 1970, Military Theory and Practice in the Age of Xenophon, Berkeley.

Anselm S., 2004, Struktur und Transparenz: Eine literaturwissenschaftliche Analyse der Feldherrnviten des Cornelius Nepos, Stuttgart.

Appel H., 2011, 'O adfectatores regni i manipulowaniu historią', Klio: Czasopismo poświęcone dziejom Polski i Powszechnym 19, pp. 3-26, https://doi. org/10.12775/KLIO.2011.055.

Arnaud-Lindet M.-P., 1993, Lucius Ampelius, Aide-mémoire, Paris, .

Arnaud-Lindet M.-P., 1997, 'Le liber Memorialis de L. Ampelius', Aufstieg und Niedergang der römischen Welt 2/34/3, pp. 2301-2312, https://doi. org/10.1515/9783110815146-009.

62 Sikorski 1966: 11-14; Lichański 2009: 101. 
Arnaud-Lindet M.-P., 1998, 'Le Liber memorialis d'Ampélius. Une propédeutique à l'étude de la rhétorique à la fin du $\mathrm{II}^{\mathrm{e}} \mathrm{s}$. de notre ère', [in:] Actes $d u$ Congrès International Quintiliano. Madrid-Calahorra, nov. 199: Quintiliano: Historia y actualidad de la rhetorica, Vol. II, pp. 825-838.

Archibald Z., 1998, The Odrysian Kingdom of Thrace: Orpheus Unmasked, Oxford.

Bettali M., 2013, Mercenari: Il mestiere delle armi nel mondo greco antico, Roma.

Bianco E., 1997, 'Ificrate, ’’́ 22, pp. 179-207.

Bianco E., 2010, 'The Third Book of Polyaenus and Ephorus', [in:] Polyainos: Neue Studien, a cura di K. Brodersen, Berlin, pp. 69-84.

Bianco E., 2011, 'Xenophon and the Tradition on the Strategoi in Fourth-Century Athens', Historika: Studi di storia greca e romana 1, pp. 39-60.

Borowska M., 2003, 'Wstęp i opracowanie', [in:] Poliajnos, Podstępy Wojenne, Warszawa.

Bugaeva N.V., 2011, 'Jeszczo raz k woprosu o żanrie, adriesatie i dostowiernosti „Pamiatnoj knigi” L. Ampielija’, Wiestnik driewniej istori 1, pp. 93-105.

Burliga B., 2016, 'Wstęp i opracowanie', [in:] Frontyn, Podstępy Wojenne, Wrocław.

Coppini D., 2008, 'Marullo Tarcaniota, Michele', [in:] Dizionario Biografico degli Italiani 71, http://www.treccani.it/enciclopedia/michele-marullo-tarcaniota_(Dizionario-Biografico)/ (ultimo accesso: 12 novembre 2019).

Cargill J., 1981, The Second Athenian League: Empire or Free Alliance?, Berkeley-Los Angeles.

Cawkwell G.L., 1962, 'Notes on the Social War', Classica et Mediaevalia 23, pp. 34-49.

Develin R., 1985, ‘Age Qualifications for Athenian Magistrates', Zeitschrift für Papyrologie und Epigraphik 61, pp. 149-159.

Ducos M., 1989, 'Ampelius (Lucius)', [in:] R. Goulet (a cura di), Dictionnaire des philosophes antiques, Vol. 1, Paris, pp. 171-173.

Duszyński W., 2016, 'Thrasybulus' Campaign in the Aegean (390-389) and Beginning of Chabrias' Military Career', Journal of Classical Studies Matica Srpska 18, pp. 71-82.

Galdi M., 1922, L'Epitome nella letteratura latina, Napoli.

Ferenc M., 2015, 'Wstęp', [in:] Stanisław Sarnicki, Księgi Hetmańskie, Kraków, pp. VII-XVII.

Habicht Ch., 1998, Hellenistic Athens, Athens. 
Hansen M.H., 1975, Eisangelia: The Sovereignty of the People's Court in Athens in the Fourth Century B.C. and the Impeachment of Generals and Politicians, Odense.

Hansen M.H., 2006, Studies in the Population of Aigina, Athens and Eretria, Copenhagen.

Harris E.M., 1989, 'Iphicrates at the Court of Cotys', The American Journal of Philology 110, pp. 264-271, https://doi.org/10.2307/295177.

Hägg T., 2012, The Art of Biography in Antiquity, Cambridge, https://doi. org/10.1017/CBO9781139061322.

Heskel J., 1997, The North Aegean Wars, 371-360 B.C., Stuttgart.

Jansen M., 2009, 'Epigramma cultum and the Anthologia Palatina: Case Studies from Michael Marullus' Epigrammata', [in:] The Neo-Latin Epigram: A Learned and Witty Genre, a cura di S. de Beer, K. A. E. Enenkel, D. Rijser, Leuven, pp. 215-231.

Kahrstedt U., 1916, 'Iphikrates', [in:] Paulys Realencyclopädie der classischen Altertumswissenschaft, vol. IX, Stuttgart, col. 2019-2021.

Kallet M., 1983, 'Iphicrates, Timotheos and Athens 371-360 B.C.', Greek, Roman, and Byzantine Studies 24, pp. 239-252.

Kirchner J., 1901, Prosopographia Attica, vol. II, Berlin.

Kołoczek B.J., 2016a, 'Wstęp', [in:] Lucjusz Ampeliusz, Repetytorium, czyli co mały Rzymianin powinien wiedzieć o świecie (Liber memorialis), Poznań, pp. 7-31.

Kołoczek B.J., 2016b, 'Liber Memorialis Lucjusza Ampeliusza - rzymski podręcznik z II w. po Chr.', Opinie Edukacyjne Polskiej Akademii Umiejętności: Prace Komisji PAU do Oceny Podręczników Szkolnych 14, pp. 47-59.

Kołoczek B.J., 2019, Historia w rzymskiej literaturze erudycyjnej: Od Warrona do Kassjodora, Kraków.

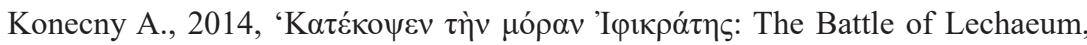
Early Summer, 390 B.C.', [in:] Iphicrates, Peltasts, and Lechaeum, a cura di N.V. Sekunda, B. Burliga, Gdańsk, pp. 7-48.

König I., 2010, Lucius Ampelius, Was ein junger Römer wissen soll, Darmstadt 2011.

Lichański J.Z., 2009, 'Aemulatio \& Imitatio - i armaty: Księgi hetmańskie Stanisława Sarnickiego a filologia', [in:] Aemulatio \& Imitatio: Powrót pisarzy starożytnych w epoce renesansu, a cura di K. Rzepkowski, Warszawa, pp. 101-112.

Lengauer W., 1979, Greek Commanders in the $5^{\text {th }}$ and $4^{\text {th }}$ Centuries B. C. Politics and Ideology: A Study of Militarism, Warszawa. 
Lengauer W., 2002, 'La qualification d'âge pour les membres de la boule athénienne', Antiquitas 26, pp. 43-50.

Lengauer W., 2004, 'Wstęp i komentarz', [in:] Ajschines, Mowy, Warszawa.

Marschall P.K., 1977, The Manuscript Tradition of Cornelius Nepos, London.

Moreno Hernandez J.J., 2002, 'Ificrates y la Fanteria Ligera Griega', Polis 14, pp. 197-224.

Parke H.W., 1933, Greek Meercenary Soldier: From the Earliest Times to the Battle of Ipsus, Oxford.

Pecorella Longo Ch., 1971, "Eterie” e gruppi politici nell'Atene del IV sec. a.C., Firenze.

Pritchett W.K., 1974, The Greek State at War, Vol. II, Berkeley.

Rehdantz K., 1845, Vitae Iphicratis Chabriae Timothei Atheniensium, Berolini

Roberts J.T., 1982, Accountability in Athenian Government, Madison.

Sage M.M., 1978, 'The "De Viris Illustribus": Chronology and Structure', Transactions of the American Philological Association 108, pp. 217-241, https:// doi.org/10.2307/284249.

Sekunda N., 1994, 'Iphicrates the Athenian and the Menestheid Family of Miletus', The Annual of the British School at Athens 89, pp. 303-306, https://doi. org/10.1017/S0068245400015434.

Sekunda N., 2014, 'The Chronology of the Iphicratean Peltast Reform', [in:] Iphicrates, Peltasts, and Lechaeum, a cura di N. V. Sekunda, B. Burliga, Gdańsk, pp. 126-144.

Sekunda N.V., 2019, 'The lochagoi of Iphicrates: Forming a Mercenary Army in the Fourth Century B.C.', [in:] War, Warlords and Interstate Relations in the Ancient Mediterranean, a cura di T. Nãco del Hoyo, F. Lopez Sanchez, pp. 6488, https://doi.org/10.1163/9789004354050_006.

Sikorski J., 1966, ‘,Księgi hetmańskie” Stanisława Sarnickiego na tle piśmiennictwa wojskowego w Polsce XVI wieku, część 1', Studia i materiały do Historii Wojskowości 12, pp. 3-69.

Sikorski J., 1967, ‘ „Księgi hetmańskie” Stanisława Sarnickiego na tle piśmiennictwa wojskowego w Polsce XVI wieku, część 2', Studia i materiaty do Historii Wojskowości 13, pp. 3-62.

Sears M., 2013, Athens, Thrace, and the Shaping of Athenian Leadership, Cambridge, https://doi.org/10.1017/CBO9781139343817.

Sprawski S., 2011, 'Cicero, the Philippicae and Philip son of Amyntas', Classica Cracoviensia 14, pp. 287-299. 
Stylianou P.J., 1998, A Historical Commentary on Diodorus Siculus Book 15, Oxford.

Terzaghi N., 1947, 'Ampeliana', Studi Italiani di Filologia Classica 21, pp. 79-92.

Toalster D., 2011, Unzeitgemässe Feldherren der Hipparch als Prototyp des erfolgreichen Feldherrn in Xenophons Hellenika, Computus.

Tuplin Ch., 1993, The Failings of Empire: A Reading of Xenophon Hellenica 2.3.11-7.5.27, Stuttgart 1993.

Westlake H.D., 1969, Essays on the Greek Historians and Greek History, New York.

Wölfflin von E., 1854, De Lucii Ampelii libro memoriali quaestiones criticae et historicae, Göttingen. 